\title{
Action influences unconscious visual processing
}

\author{
Jihyun Suh ${ }^{1} \cdot$ Richard A. Abrams ${ }^{1}$
}

Published online: 26 March 2018

(C) The Psychonomic Society, Inc. 2018

\begin{abstract}
It has long been known that action is tightly linked to visual perception. In support of this connection, recent studies have shown that making a simple action towards a visual object can bias subsequent visual processing of features of the acted-on object. The present study examined whether conscious awareness of the acted-on object is necessary to yield this action effect. In two experiments, we found that making an action towards an invisible object resulted in greater priming in a subsequent visual search task. This shows that conscious awareness is not necessary to obtain the action-induced visual bias. More importantly, the result implies that action might amplify the sensory signal from the subliminally presented object, which is presumed to occur during early visual processing.
\end{abstract}

Keywords Perception and Action $\cdot$ Visual perception $\cdot$ Visual awareness

\section{Introduction}

Recent studies have revealed that actions can influence visual perception. For example, Bekering and Neggers (2002) showed that planning an action towards a visual object can influence how features of the object are preferentially processed. In their experiment, participants were asked to either grasp or point at a graspable object defined by color and orientation amongst distractor objects. When a grasping action was planned, participants were better able to ignore non-target orientations, presumably because orientation was an important feature for performing the planned grasping action. Similarly, studies have shown that a planned grasping movement enhanced people's ability to judge the size of a visual stimulus compared to a planned pointing movement (Wykowska, Schubo, \& Hommel, 2009), and a planned power grasp increased visual sensitivity to relatively big changes, whereas a planned precision grasp increased visual sensitivity to relatively small changes (Symes, Tucker, Ellis, Vainio, \& Ottobani, 2008), implying that the visual system prefers visual features most relevant to a planned action.

Jihyun Suh

jihyun.suh@wustl.edu

1 Psychological and Brain Sciences, Washington University, St. Louis, MO, USA
More recent evidence has suggested that not only a functional action (e.g., grasping or pointing), but also a simple arbitrary action can influence how the visual system selectively processes information. Specifically, several studies have reported that simply pressing a key in response to a visual object leads to a strong perceptual bias towards the features of the acted-on object (Buttacio \& Hahn, 2011; Weidler \& Abrams, 2014, 2017). For instance, in Buttacio and Hahn (2011), participants were asked to either press a key to a colored circle (the prime) or to do nothing but view the circle. Then, a visual search task followed in which a target or distractor was rendered in the prime color. The results showed that the previously seen color (the color of the prime) was preferentially processed resulting in a faster response when the target was the prime color but a slower response when the distractor was the prime color. However, importantly, this pattern was observed only when participants had made a key press response to the prime. This selective bias towards the features of an acted-on object has been referred to as the action effect. Several studies have replicated and extended the basic findings. For example, features of previously acted-on objects are prioritized in a pop-out search in which the target is perceptually salient so that a serial search is not required (Weidler \& Abrams, 2017). Furthermore, acting to the prime also biases eye movements during search toward the features of the actedon object (Wang, Sun, Sun, Weidler, \& Abrams, 2017).

Although a number of studies have provided evidence supporting the role of action in selective visual processing, the underlying mechanism remains in question. Buttacio and 
Hahn (2011) suggested that making an action might strengthen the visual representation of the acted-on object's features, which they referred to as the trace of the object. According to this view, when an action is made to a red prime, for example, the intensity of the visual representation of the perceived color red would become stronger compared to when the prime is passively viewed. Therefore, subsequent visual perception is more likely to be guided by the features that are compatible with the trace after an action.

However, the nature of the trace has not been clearly defined. There are two broad possibilities. First, it is possible that action might strengthen the episodic trace of the events associated with the action (e.g., an event involving pressing a key to an object of a specific color). According to this view, the action effect is a consequence of richer episodic processing of the prime object when it is accompanied by an action. To be clear, we assume that episodic processing ${ }^{1}$ means the processing of a personal event with an engagement of conscious awareness. Therefore, if the action effect occurs mainly through the enhancement of episodic information, conscious awareness would be necessary for it to occur. Alternatively, it is also possible that action might strengthen the sensory trace of the prime object early in visual processing (e.g., V1), essentially causing the prime object to be more salient. In this case, conscious awareness would not be necessary for action to influence sensory information about the primed object.

Based on the available evidence, however, it is not possible to distinguish between the episodic enhancement and the sensory enhancement explanations of the action effect because in all past experiments the prime object was always presented with full awareness. In the present study, we sought to learn more about the locus of the action effect by presenting the prime object subliminally. As a result, episodic information regarding the prime color would not be available, but early sensory enhancement of the prime object would still be possible.

In order to present the prime color unconsciously, we adopted the continuous flash suppression (CFS) technique (Tsuchiya \& Koch, 2005) that is widely used to present visual stimuli subliminally. In a typical experiment using CFS, stimuli are presented dichoptically, with different images projected to each eye. Critically, a visually salient mosaic patch flickers continuously in the dominant eye to dominate visual awareness while blocking awareness of the image in the nondominant (suppressed) eye. Besides the CFS manipulation, the present study used the typical action effect paradigm as in Weidler and Abrams' (2014) Experiment 3. In the present study, participants were requested to make an action only following a "go" signal (i.e., the word "Go" in Experiment 1 and an auditory tone in Experiment 2) but do nothing following a "no" signal (i.e., the word "No" in Experiment 1 and an

\footnotetext{
$\overline{1 \text { Tulving (2002) }}$ identifies an important feature of episodic memory as "conscious awareness of what had happened in the past" (p. 4).
}

auditory tone in Experiment 2). However, unlike in the original study, here the prime object was presented on the suppressed eye. As a result, participants were not consciously aware of the prime color while they were making the action or viewing the prime.

We hypothesized that if an action strengthens an early sensory trace, there should be a greater priming effect following an action compared to following passive viewing of the prime even when the prime is invisible (i.e., the action effect would occur even with invisible primes). However, if action strengthens an episodic trace of the prime object, then conscious awareness of the prime would be necessary, and there will be no effect of action on the priming effect with invisible primes.

\section{Experiment 1}

The goal of Experiment 1 was to determine whether an action effect occurs for an unconsciously presented color. Participants were asked to press the spacebar when the word "Go" was seen but do nothing when the word "No" was seen on the dominant eye where the flickering mosaic patch was presented. While the go/no word was present to the dominant eye, a colored disk (the prime) was presented to the suppressed eye - but participants were not able to consciously perceive it. A visual search task followed in which the prime color appeared as a feature of the target or as a feature of the distractor.

\section{Method}

Participants Twenty-four students from Washington University in St. Louis, MO, participated to fulfill a partial requirement for course credit. This is the same number of participants that was used in Weidler and Abrams (2014). All participants had normal or corrected-to-normal vision and normal color vision. Each participant reported the dominant eye using a conventional dominant eye test (i.e., hand reaching test). Participants were given a written informed consent form and agreed to the procedures approved by the Institutional Review Board of Washington University in St. Louis.

Apparatus The experiment was programmed in Python using Psychopy (Peirce, 2007). Stimuli were presented on the left and right sides of a 20-in. CRT monitor. An adjustable mirror stereoscope was located $36 \mathrm{~cm}$ from the monitor, and ensured that each eye had a view of only one-half of the display.

Stimuli All stimuli were presented in a dark gray (CIE color coordinates: L:82.4, a:0, b:1.1) square region surrounded by a light gray frame (L:90.9, a: -.4, b: $1,18^{\circ} \times 18^{\circ}$, frame width: $1.5^{\circ}$ ). The CFS stimulus (i.e., the flickering mosaic; $15^{\circ} \times 15^{\circ}$, 
refresh rate: $42.5 \mathrm{~Hz}$ ) was generated by randomly selecting colors (red, L: 60.0, a: 52.7, b: 51.5; green, L: 65.0, a: -65.1, b: 56.5; blue, L: 52.7, a:14.0, b: -59.1; yellow, L: 78.9, a: -13.5, b: 31.5 ; and black) for each cell of a $17 \times 17$ matrix and reselecting at every screen refresh. A color circle (13.6 ${ }^{\circ}$; red, green, blue, or yellow) served as the prime object. A Gaussian mask was applied to the circle to blur its border. Also, a layer of gray color at $50 \%$ opacity was overlaid on the prime to ensure that it was not salient enough to break the interocular suppression.

Procedure Each trial contained three parts: action task, visual search task, and awareness report.

Action task The CFS stimulus was always presented to the participant's dominant eye while the color prime was presented to the suppressed eye. Participants were not informed about the prime. The sequence of events on a typical trial is shown in Fig. 1. Each trial began with a blank screen for 1s (not shown in the figure). In the suppressed eye, following a blank screen of $494 \mathrm{~ms}$, the color prime faded in for $494 \mathrm{~ms}$ until it reached full contrast. The prime color was randomly selected from red, green, blue, and yellow with an equal probability. The color prime remained at full contrast for $1,482 \mathrm{~ms}$ and then faded out for another $494 \mathrm{~ms}$. In the dominant eye, the CFS stimulus was continuously shown for $3,458 \mathrm{~ms}$ while the color prime was being presented (to the suppressed eye). After 1,729 ms from CFS onset, "Go" or "No" (font size: $1.4^{\circ}$ ) was presented superimposed on the CFS patch for $1 \mathrm{~s}$. Participants were asked to press the spacebar with the left hand as soon as they saw the word "Go" (producing an action trial) but do nothing for the word "No" (yielding a viewing trial). The word always appeared while the color prime was shown at full contrast.

Visual search task Immediately after the action task, a visual search task followed. During the search task, all stimuli were presented to both dominant and suppressed eyes. The search task began with a white fixation cross $\left(0.9^{\circ} \times 0.9^{\circ}\right)$ at the center of the box for $1 \mathrm{~s}$. Then, two colored circles $\left(3.7^{\circ}\right)$ were presented - one on each side of fixation $\left(3.6^{\circ}\right.$ from the center). One of the circles contained a tilted line $\left(2.6^{\circ} \times 0.1^{\circ}, 4^{\circ}\right.$ or $-4^{\circ}$ from vertical) and the other circle contained a vertical line. Participants were asked to press the left or right arrow key with the right hand as quickly and accurately as possible according to the orientation of the tilted line. Examples of search displays following the action and viewing conditions are illustrated in Fig. 2. The search display always included the prime color but the target (tilted line) was embedded in the prime color on one-half of the trials (valid), whereas the distractor (vertical line) was embedded in the prime color on the other
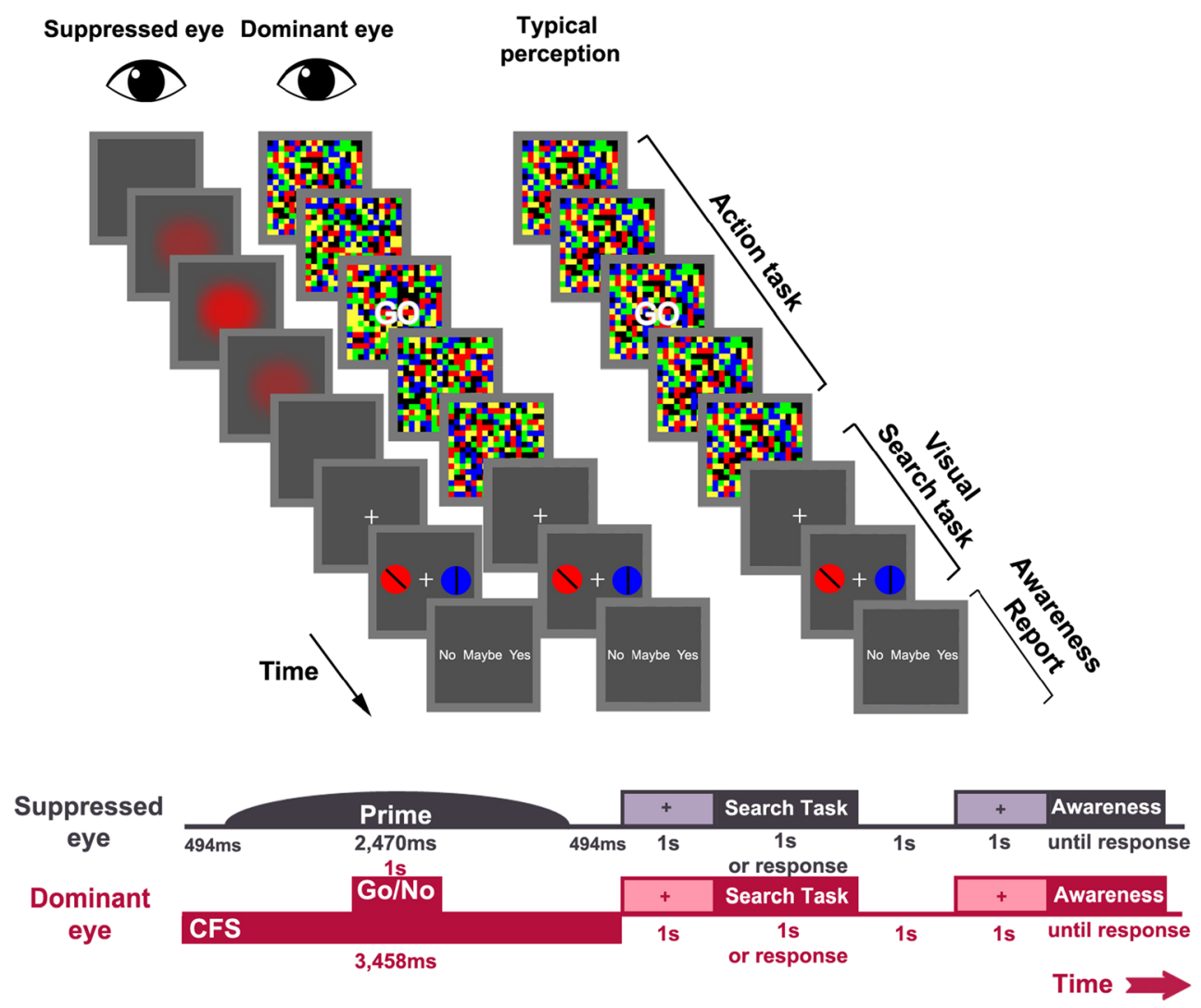

Fig. 1 Sequence of events on a trial in Experiment 1 


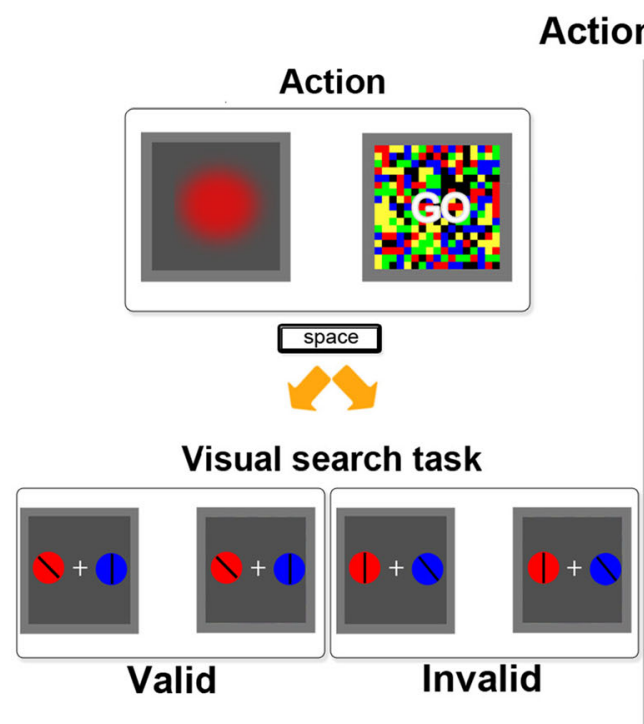

Fig. 2 Examples of the visual displays for each of the experimental conditions in Experiment 1. The left and right panels in each frame depict the stimuli presented to the suppressed and dominant eyes, respectively. The prime color was randomly presented from a color list (red, green, blue, yellow). For the valid condition, the target was

half of the trials (invalid). An error message ("incorrect") was given to incorrect responses for $1 \mathrm{~s}$ with a brief auditory tone (200 Hz, $500 \mathrm{~ms})$.

Awareness report In order to confirm that the prime object was not consciously perceived, participants were asked at the end of each trial to report whether they had seen anything other than the CFS during the action task. Participants were given three options ("Yes," "No," and "Maybe") and asked to select one based on their subjective visual experience. There was no time limit for the awareness report.

Design Each participant completed 12 practice trials and four blocks of 48 test trials. A set of 2 (go vs. no) $\times 2$ (valid vs. invalid $) \times 4$ (prime colors $) \times 2$ (target orientation; $4^{\circ}$ vs. $\left.-4^{\circ}\right) \times$ 2 (target position; left vs. right) trials was repeated three times yielding the total of 192 test trials, which were presented in a random order. The target color for invalid trials and distractor color for valid trials were chosen from the remaining three non-prime colors.

\section{Results}

Table 1 presents the mean percentage of each response for the awareness report showing that CFS successfully blocked the conscious awareness of the suppressed color prime. One participant was excluded because the awareness report showed that he or she perceived the prime color (i.e., selected "yes" or "maybe" for the awareness report) on the majority of trials (83.35\%; more than three standard deviations (SDs) above the mean rate). Another participant was excluded due to a high
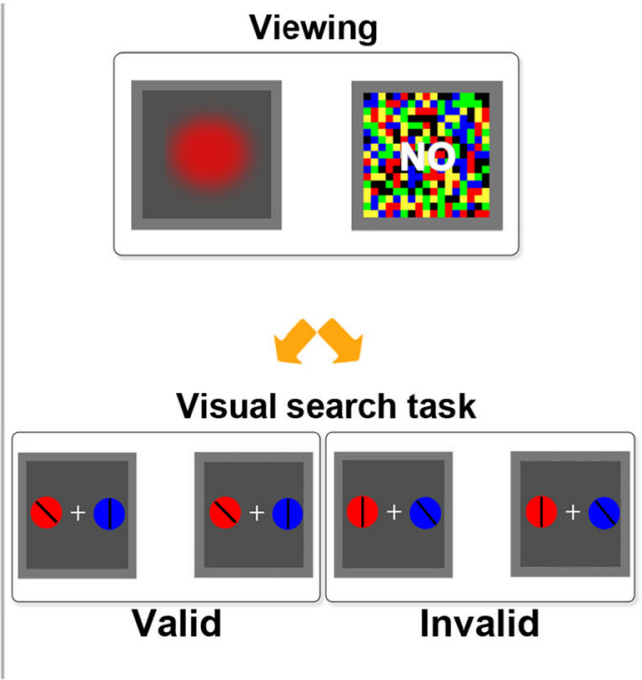

embedded in the prime color in the search task. For the invalid condition, the target was embedded in a non-primed color but the distractor was embedded in the prime color. "Space" designates an action (spacebar press)

error rate during the visual search task (44.1\%; more than three SDs above the mean). For the remaining data, trials where participants selected "yes" or "maybe" for the awareness report were excluded as well as trials where participants did not make a choice (no response), resulting in elimination of $5.8 \%$ of trials.

Action task The overall accuracy for the action task was $97.23 \%$ and average reaction time (RT) was $695 \mathrm{~ms}$. Participants were more accurate on the action trials $(98.67 \%)$ than the viewing trials $(95.79 \%), t(21)=3.465, p=.002, d=0.74$.

\section{Visual search task}

Reaction time Trials where the search task RT was more than three SDs from each individual's mean and those containing incorrect responses (either during the action task or the visual search task) were considered as errors and excluded from analysis $(9.57 \%)$. The overall mean RT was $650 \mathrm{~ms}$. A repeated

Table 1. Mean percentage of each response in the awareness report (\%) from Experiment 1 and Experiment 2

\begin{tabular}{llll}
\hline & Experiment 1 & \multicolumn{2}{l}{ Experiment 2 } \\
\cline { 3 - 4 } & & Suppressed & Catch \\
\hline Yes & 1.82 & 3.48 & 94.32 \\
Maybe & 1.3 & 1.99 & 0.45 \\
No & 94.46 & 94.53 & 5.23 \\
No response & 2.41 & - & - \\
\hline
\end{tabular}


measures ANOVA was conducted as a function of trial type (valid vs. invalid) and action (action vs. viewing). The data are plotted in Fig. 3. Overall, participants were slightly slower for invalid than valid trials but this difference was not significant, $F(1,21)=3.727, p=.067, \eta_{\mathrm{p}}^{2}=.15$. Responses were significantly slower overall for action trials compared to viewing trials, $F(1,21)=6.7, p=.017, \eta_{\mathrm{p}}^{2}=24$. Importantly, a significant two-way interaction was observed, indicating that the priming effect (invalid - valid) was greater for the action than the viewing condition, $F(1,21)=5.86, p=.025, \eta_{\mathrm{p}}^{2}=.22$, which shows the typical action effect. ${ }^{2}$ A planned paired samples t-test showed that invalid trials were significantly slower than valid trials in the action condition, where participants previously acted toward the prime, $t(21)=2.584, p=.017, d=$ 0.55 , However, this difference was not significant in the viewing condition, $t(21)=1.064, p=.299, d=0.23$.

Accuracy The search accuracy was computed by considering only trials that contained correct action responses (trials where participants pressed the spacebar in response to a "No" cue or did not respond to a "Go" cue were excluded, resulting in elimination of $2.77 \%$ of trials). The values are summarized in Table 2. The overall error rate was $6.93 \%$. The main effect of action, $F(1,21)=1.75, p=.2, \eta_{\mathrm{p}}^{2}=08$, priming, $F(1,21)=$ $2.996, p=.098, \eta_{p}^{2}=13$, and the interaction, $F(1,21)=1.228$, $p=.28, \eta_{\mathrm{p}}^{2}=06$, were not significant.

\section{Discussion}

The present experiment showed that a simple action enhanced the effect of a color prime even when the color prime was not consciously presented. Given the fact that participants did not consciously perceive the prime color, the result suggests that the action might have amplified the sensory signal from the suppressed eye, as opposed to having strengthened an episodic trace of the presentation of the prime.

Despite this surprising finding, it is worth considering the participants' awareness reports. Even though we excluded all trials on which the participant reported any awareness of the prime, it is still possible that on some trials participants perceived at least part of the prime, but chose not to report it. This seems possible especially if the participant never perceived the entire prime and thus would not have a sense of what the prime object looked like. We addressed this concern in Experiment 2.

\footnotetext{
${ }^{2}$ An additional analysis was conducted to rule out the possibility that pressing the spacebar with the left hand caused an attentional bias to the left side of the display, possibly resulting in a greater priming effect (invalid-valid) when the target was presented on the left side during the visual search task. A repeated measures ANOVA with factors of target location (left vs. right), action (action vs. viewing) and validity (valid vs. invalid) revealed neither a main effect of the target location nor was target location involved in any two-way or threeway interaction, all $F_{\mathbf{S}}<1$, confirming that the action did not simply prime the spatial dimension.
}

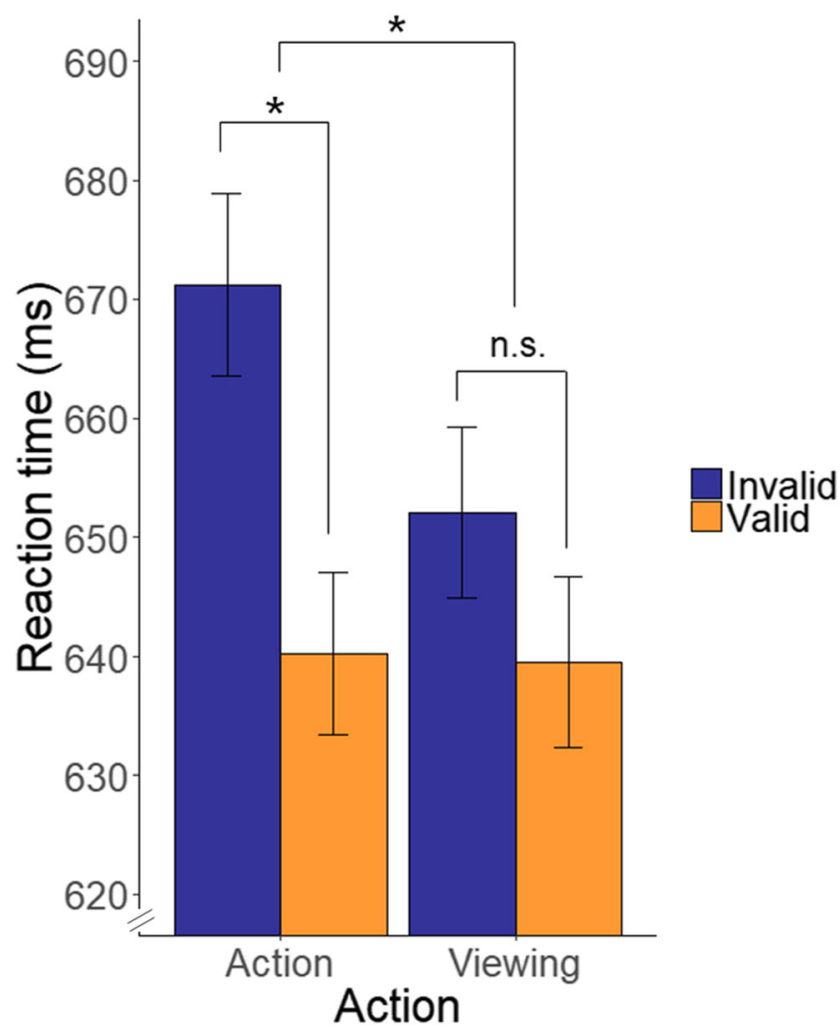

Fig. 3 Visual search reaction time for the visual search task in Experiment 1. Error bars depict within-subject standard errors. *: $\mathrm{p}<.05$

\section{Experiment 2}

The main purpose of Experiment 2 was to replicate Experiment 1, with two changes designed to increase confidence in the effect. First, it is possible that many participants in Experiment 1 rarely or never saw the full prime, and as a result they were unmotivated to report awareness of the prime even if they had seen a portion of it (Yang, Brascamp, Kang, \& Blake, 2014). In Experiment 2, to solve this issue, catch trials, on which the prime should have been consciously perceived, were added to create familiarity with the prime and to create a scenario in which prime reports were expected. The catch trials also allowed us to gauge each participant's willingness to indicate awareness of the prime color. Second, unlike the

Table 2. Mean percentage of error (\%) in the visual search task from Experiment 1 and Experiment 2

\begin{tabular}{|c|c|c|c|c|}
\hline & \multicolumn{2}{|l|}{ Action } & \multicolumn{2}{|c|}{ Viewing } \\
\hline & Invalid & Valid & Invalid & Valid \\
\hline Experiment 1 & 8.09 & 6.96 & 7.8 & 4.89 \\
\hline $\begin{array}{l}\text { Experiment } 2: \\
\text { suppressed trials }\end{array}$ & 2.33 & 5.17 & 3.32 & 3.07 \\
\hline $\begin{array}{l}\text { Experiment 2: } \\
\text { catch trials }\end{array}$ & 4.65 & 0 & 4.8 & 5.3 \\
\hline
\end{tabular}


typical action effect paradigm where the go/no signal and prime color are presented in a sequential order, the go/no signal (the word "Go"/"No") and the subliminal prime color were presented simultaneously in Experiment 1 . Therefore, it is possible that the presence of the word might have diminished the processing of the prime, and as a result our findings may underestimate the true magnitude of the priming effect. Therefore, in Experiment 2, the pitch of an audible tone was used to signal whether an action was required instead of a visible "Go" or "No" word.

\section{Method}

Participants Twenty-four students from Washington University in St. Louis, MO, participated to fulfill a partial requirement for course credit. All of them had normal or corrected-to-normal vision and normal color vision. Participants were given a written informed consent form and agreed upon the procedures approved by the IRB of Washington University in St. Louis.

Apparatus, stimuli, and procedure This experiment was very similar to Experiment 1. However, instead of a word cue for the action task, an auditory tone $(400 \mathrm{~Hz}$ or $800 \mathrm{~Hz}$ ) was presented for $200 \mathrm{~ms}$. Participants were asked to press the spacebar as soon as the pitch signaling an action was presented - for one-half of the participants that was the lower pitch, for the others it was the higher pitch. For incorrect action responses, a feedback message was presented on the screen for $500 \mathrm{~ms}$ ("incorrect," font size: $\left.1.3^{\circ}\right)$. In addition to the suppressed trials in which the prime object was shown only to the non-dominant eye preventing conscious awareness of the prime color, catch trials were added where the prime was presented to both non-dominant and dominant eyes. In the dominant eye, the prime color was superimposed on the CFS stimulus resulting in conscious awareness of the prime. During the awareness report, unlike in Experiment 1, which permitted no response, participants were required to select one of three options ("Yes," "No," or "Maybe") to continue.

Design Each participant completed 16 practice trials and four blocks of 48 test trials. As in Experiment 1, a set of 2 (go vs. no) $\times 2$ (valid vs. invalid) $\times 4$ (prime colors $) \times$ 2 (target orientation; $4^{\circ}$ vs. $-4^{\circ}$ ) $\times 2$ (target position; left vs. right) trials was repeated three times yielding a total of 192 test trials. In addition, 40 catch trials were added, presented randomly during the session. For the catch trials, each prime color (four colors) was presented ten times but other conditions (go vs. no, valid vs. invalid, target orientation, and target position) were randomly selected for each trial.

\section{Results}

Two participants were excluded from analysis. One participant reported seeing the prime color (i.e., selected "yes" or "maybe" in the awareness report) during the action task on the majority of the trials $(85.93 \%$; more than three SDs above the mean awareness rate). The other participant was excluded due to a high error rate in the visual search task (49.91\%; more than three SDs above the mean). After excluding those two participants, those remaining reported "yes" or "maybe" in the awareness report in $5.5 \%$ of trials and those trials were excluded from further analysis (see Table 1). The percentage of "yes" or "maybe" responses was numerically greater than that of Experiment $1(3.1 \%)$ but failed to reach statistical significance, $t(42)=1.156, p=.254, d=0.35$.

Action task The overall accuracy for the action task was $97 \%$. Participants were equally accurate in the action condition $(97.22 \%)$ and the viewing condition $(96.98 \%), t(21)=.246, p$ $=.808, d=0.05$. For the action condition, the mean RT was $917 \mathrm{~ms}$.

Visual search task Trials with RTs more than three SDs from each individual's mean, and incorrect trials (i.e., trials on which an error occurred in either the action task or the visual search task) were excluded from analysis (7.55\%).

\section{Suppressed trials}

Reaction time Search task RTs for the suppressed trials are shown in Fig. 4 (left panel). A repeated measures ANOVA was conducted as a function of trial type (valid vs. invalid) and action (action vs. viewing). Neither the main effect of trial type nor of action was significant $\left(F_{S}<1\right)$. However, importantly, the interaction was significant, showing that the magnitude of the priming effect was greater after an action than after viewing, $F(1,21)=11.17, p=.003, \eta_{\mathrm{p}}^{2}=.35$, replicating the results of Experiment 1 and revealing an action effect for subliminally presented primes. ${ }^{3} \mathrm{~A}$ planned comparison showed that the priming effect was significant for the action condition $(t(21)=2.107, p=.047, d=0.45)$, but not for the viewing condition $(t(21)=1.143, p=.266, d=0.24)$.

Accuracy Trials with incorrect go/no responses were eliminated in calculating the error rate during the search task $(2.82 \%)$. The error rates for each condition are described in Table 2. A repeated measures ANOVA with trial type and action revealed that the main effect of action was significant, $F(1,21)=8.057$,

\footnotetext{
${ }^{3}$ As in Experiment 1, a repeated measures ANOVA with factors of target location (left vs. right), action (action vs. viewing) and validity (valid vs. invalid) revealed neither a main effect of target location nor any two-way or three-way interaction involving target location $\left(F_{\mathrm{S}}<1\right)$, confirming that action did not simply prime the spatial dimension.
} 


\section{Suppressed trials}

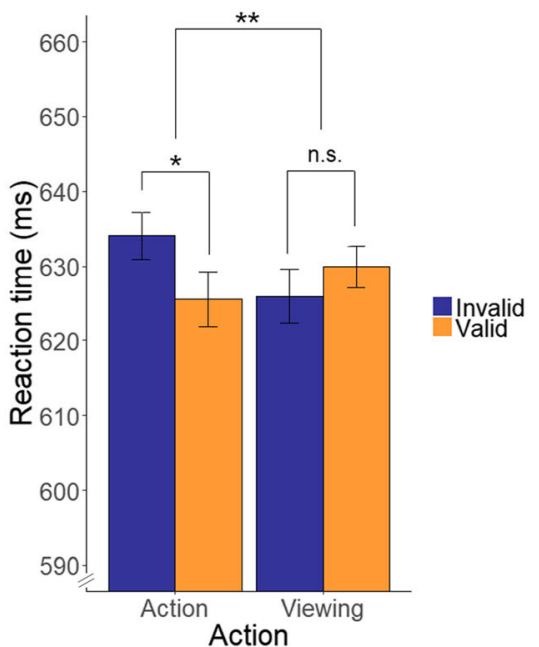

\section{Catch trials}

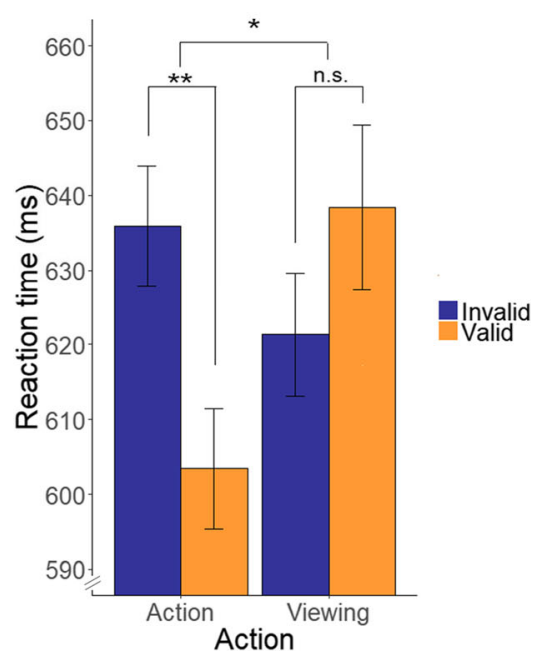

Fig. 4 Visual search reaction time in Experiment 2. Left: suppressed trials. Right: catch trials. Error bars depict within-subject standard errors. **: $\mathrm{p}<$ $.01, *: \mathrm{p}<.05$

$p=.010, \eta_{\mathrm{p}}^{2}=.28$ indicating that participants made more errors when they had previously made an action. However, the main effect of trial type $(F<1)$ and interaction $(F(1,21)=$ $\left.1.153, p=.295, \eta_{\mathrm{p}}^{2}=.05\right)$ were not significant. ${ }^{4}$

Catch trials The same repeated measures ANOVA was conducted for the catch trials, on which the participants were consciously aware of the prime color, as in all previous action effect experiments (e.g., Weidler \& Abrams, 2014, 2017). Trials on which participants responded "no" to the awareness report were considered as errors and excluded from analysis $(4.43 \%)$.

Reaction time The RT data are plotted in Fig. 4 (right panel). Repeated measures ANOVA revealed that neither the main effect of action $\left(F(1,21)=1.051, p=.317, \eta_{\mathrm{p}}^{2}=.05\right)$ nor of trial type $\left(F(1,21)=1.16, p=.294, \eta_{\mathrm{p}}^{2}=.05\right)$ was significant. However, there was a significant interaction, $F(1,21)=$ $6.949, p=.015, \eta_{\mathrm{p}}^{2}=.25$, showing that the priming effect was greater after an action compared to viewing, revealing the

\footnotetext{
${ }^{4}$ Despite the fact that there was not a significant interaction involving the error rates, there was numerically a trend toward a speed-accuracy tradeoff. As a result, one might wish to convert the RTs and errors into a single index such as the inverse efficiency score (IES), which is a combined measure of RT and proportion of errors (PE) originally proposed by Townsend and Ashby (1978, 1983). However, it has been argued (Bruyer \& Brysbaert, 2011) that such a conversion is meaningful only when RT and PE are positively correlated. In the present experiment, there was no such correlation $(r(20)=.003, p=.99)$. Additionally, such a conversion adds noise, reducing the power of the comparison (Bruyer \& Brysbaert, 2011). Nevertheless, with these caveats in mind, we did perform the conversion. The IES continued to reveal the same pattern as before: a greater validity effect for the suppressed action trials compared to the suppressed viewing trials, although the interaction was not statistically significant, presumably due to the introduction of noise by the conversion process.
}

typical action effect. Paired t-tests also revealed that the priming effect was significant for the action condition $(t(21)=$ $3.334, p=.003, d=0.71)$, but not for the viewing condition $(t(21)=1.256, p=.223, d=0.27)$.

Accuracy Again, trials with incorrect go/no responses were eliminated for the analysis of error rate $(4.43 \%)$. The data are shown in Table 2 . The main effect of action $(F(1,21)=$ 4.23, $\left.p=.13, \eta_{\mathrm{p}}^{2}=.10\right)$ shows that participants were more accurate on the visual search task for action compared to viewing. The main effect of trial type was not significant, $F(1,21)=1.945, p=.178, \eta_{\mathrm{p}}^{2}=.09$. However, there was a marginal interaction $\left(F(1,21)=3.116, p=.092, \eta_{\mathrm{p}}^{2}=.13\right)$ showing the typical action effect.

A planned comparison revealed that participants made more errors for invalid than valid trials following an action, $t(21)=$ $3.129, p=.005, d=0.67$. This difference was not meaningful for the viewing condition, $t(21)=.199, p=.844, d=0.40$.

In addition, an analysis of the overall data. including both suppressed and non-suppressed trials, revealed a marginally significant three-way interaction between trial type (valid vs. invalid), action (action vs. viewing), and visual awareness (suppressed vs. catch trials), $F(1,21)=4.099, p=.056, \eta_{p}^{2}$ $=.16$, indicating the tendency for action's effect on color priming to be greater when participants were consciously aware of the prime color.

\section{Discussion}

In this experiment we included catch trials with a visible prime object in order to ensure that participants would be willing to report all primes that were seen on the critical trials designed to suppress conscious awareness of the primes. Importantly, 
we again found a greater color priming effect after a motor response had been made towards the prime (i.e., an action effect), even though the prime was not consciously perceived. Indeed, the effect size here $\left(\eta^{2} \mathrm{p}=.35\right.$ for the interaction) was greater than that from Experiment $1\left(\eta_{p}^{2}=.22\right)$. Additionally, we also observed the same pattern for non-suppressed trials (in which the primes were visible), but the effect size was bigger than that of the suppressed trials. This suggests that conscious processing of the prime may also play a role in producing the action effect.

\section{General discussion}

In two experiments, we observed a typical action effect - that is, a bias in visual search for the color of a previously actedupon prime object. Importantly, in the present experiments unlike in past research, the prime object was presented in a manner that prevented conscious awareness. Thus, the present result suggests that the action effect can occur even when the prime is presented outside of visual awareness. This implies that the action effect arises at least in part from early enhancement of the sensory signal from the prime caused by the action, and not exclusively due to a strengthened episodicmemory trace of the action event. This conclusion follows because the episodic strengthening would require conscious awareness of the prime, yet we found an action effect even when the prime had not been consciously seen. Additionally, Experiment 2 included catch trials on which the prime was presented so as to be consciously perceived, as in the typical action-effect paradigm. Those trials yielded color priming (i.e., the action effect) that was greater in magnitude than the priming from the trials on which conscious perception was suppressed. This finding suggests that a component of the action effect may arise from a strengthening of the episodic trace as well.

It is important to note that even though we found no effect of an unconsciously presented color when there was no action towards the prime (i.e., the viewing trials), we are not suggesting that an action is necessary for an unconscious color priming effect. Indeed, all previous demonstrations of unconscious color priming have used paradigms in which responses were never made to the unconsciously presented object (Ansorge, Kiss, \& Eimer, 2009; Ansorge \& Neumann, 2005; Breitmeyer, Ro, \& Singhal, 2004; Breitmeyer, Ro, Öĝmen, \& Todd, 2007; Jacob, Breitmeyer, \& Treviño, 2013; Hong \& Blake, 2009; Hsieh, Colas, \& Kanwisher, 2011; Ro, Singhal, Breitmeyer, \& Garcia, 2009; Railo, Salminen-Vaparanta, Henriksson, Revonsuo, \& Koivisto, 2012; Schmidt, 2000, 2002; Tapia, Breitmeyer, \& Shooner, 2010; Tsuchiya \& Koch, 2005). We assume that the many differences between our paradigm and the earlier ones account for the discrepancy.
In support of this, some previous studies have also found that the unconscious priming effect could disappear depending on task requirements. For example, Ansorge and Neumann (2005) showed that spatial priming by an invisible spatial cue disappeared when a target for a probe task was defined by a non-spatial feature (i.e., color) not by its spatial location. A similar finding was reported by Tapia et al. (2010), who found that an unconsciously primed color did not reveal priming when participants were asked to report the form of a colored geometric shape. Thus, the absence of the unconscious priming effect in our viewing trials is not an unusual finding. Instead, it highlights another key difference between the present results and those from previous studies, which is that color was a task-irrelevant feature in the present experiments, whereas it was a task-relevant feature dimension in most previous work. For example, participants were asked to report the unconsciously primed color explicitly (e.g., guess the color) or by searching for the same or a different color (Ansorge et al., 2009; Breitmeyer et al., 2004; Breitmeyer et al., 2007; Hong \& Blake, 2009; Hsieh et al., 2011; Jacob et al., 2013; Railo et al., 2012; Ro et al., 2009; Schmidt, 2000, 2002; Tsuchiya \& Koch, 2005) in the prior studies. On the other hand, our participants were asked to search for a tilted line not the color and, for the first time, unconscious color priming was observed even though the color was task-irrelevant. Therefore, our findings suggest that action's modulation of unconscious color processing was not contingent on topdown goals such as an attentional set for color.

Consistent with this, several studies have concluded that unconscious color priming is mediated by low-level sensory signals (Breitmeyer et al., 2007; Breitmeyer et al., 2004; Railo et al., 2012). For example, Breitmeyer et al. (2004, 2007) showed that unconscious color priming depended on the physical wavelength of the prime color not the subjective perception of the color. Similarly, Railo et al. (2012) showed that stimulating the visual cortex with transcranial magnetic stimulation immediately after the presentation of a masked color prime, disabling early visual processing of the unconsciously perceived color, reduced the color priming effect. Taken together, the results suggest that unconscious color priming occurs early in visual processing. Furthermore, because action modulated the magnitude of unconscious priming, it seems likely that action also exerts its effects early in processing, perhaps by enhancing sensory signals arising from the prime object.

It is the case that previous studies have shown visuo-motor interactions without conscious awareness (Lunghi, Morrone, \& Alais, 2014; Lunghi, Binda, \& Morrone, 2010; Maruya, Yang \& Blake, 2007; Mudrik, Faivre, \& Koch, 2014; Di Pace \& Saracini, 2014; Salomon, Lim, Herbelin, Hesselmann, \& Blanke, 2013), supporting the idea that action may boost the sensory signal from a suppressed visual stimulus. However, because the unconsciously primed color was not associated with a specific response in our experiments, 
we do not believe that response priming contributed to the present result.

In addition, it is worth noting that the action effect seems to have been driven by performance on the invalid trials, not the valid trials, especially in Experiment 1 . This may have been caused by general slowing on the action trials perhaps due to the requirement to make two responses on those trials (one in the action effect task and one in the search task). A similar pattern has been observed in some previous action effect experiments, but not all (compare, for example, Experiments 1 and 4 in Weidler \& Abrams, 2014).

One could argue that participants might have unconsciously accessed semantic information about the prime presented to the suppressed eye. However, recent studies have failed to find semantic priming of a stimulus presented under CFS, which reduces this possibility (Hesselmann, Darcy, Ludwig, \& Sterzer, 2016; Kang, Blake, \& Woodman, 2011; Sakuraba, Sakai, Yamanaka, Yokosawa, \& Hirayama, 2012). Based on the findings noted above, we suggest that it seems unlikely that semantic priming played a significant role in the action effect with unconsciously perceived primes.

It is also worth discussing how action-induced sensory enhancement could cause a greater priming effect in a subsequent visual search task. Soto, Mäntylä, and Silvanto (2011) showed that an unconsciously perceived visual object could remain in visual working memory for up to $5 \mathrm{~s}$. One possibility is that a simple action made toward the prime object in our experiments might have caused it to remain in visual working memory for a longer time period compared to the prime object without an action. However, to confirm this possibility, future research will be needed to learn more about temporal aspects of action's influence on unconscious visual processing.

The present study sought to reveal the underlying nature of action's influence on visual processing. The results showed that unconsciously perceived color primes produced a greater color priming effect when a simple action was made towards them, showing that an action can strengthen the sensory trace of the acted-on object without engagement of visual awareness.

\section{References}

Ansorge, U., Kiss, M., \& Eimer, M. (2009). Goal-driven attentional capture by invisible colors: Evidence from event-related potentials. Psychonomic Bulletin \& Review, 16(4), 648-653.

Ansorge, U., \& Neumann, O. (2005). Intentions determine the effect of invisible metacontrast-masked primes: Evidence for top-down contingencies in a peripheral cuing task. Journal of Experimental Psychology: Human Perception and Performance, 31(4), 762.

Bekkering, H., \& Neggers, S. F. (2002). Visual search is modulated by action intentions. Psychological Science, 13(4), 370-374.
Breitmeyer, B. G., Ro, T., Öĝmen, H., \& Todd, S. (2007). Unconscious, stimulus-dependent priming and conscious, percept-dependent priming with chromatic stimuli. Attention, Perception, \& Psychophysics, 69(4), 550-557.

Breitmeyer, B. G., Ro, T., \& Singhal, N. S. (2004). Unconscious color priming occurs at stimulus-not percept-dependent levels of processing. Psychological Science, 15(3), 198-202.

Bruyer, R., \& Brysbaert, M. (2011). Combining speed and accuracy in cognitive psychology: is the inverse efficiency score (IES) a better dependent variable than the mean reaction time (RT) and the percentage of errors (PE)? Psychologica Belgica, 51(1), 5-13.

Buttaccio, D. R., \& Hahn, S. (2011). The influence of action on visual search: Behavioral response toward stimuli modifies the selection process. Attention, Perception, \& Psychophysics, 73(5), 1453-1466.

Di Pace, E., \& Saracini, C. (2014). Action imitation changes perceptual alternations in binocular rivalry. PloS one, 9(5), e98305.

Hesselmann, G., Darcy, N., Ludwig, K., \& Sterzer, P. (2016). Priming in a shape task but not in a category task under continuous flash suppression. Journal of Vision, 16(3), 17-17.

Hong, S. W., \& Blake, R. (2009). Interocular suppression differentially affects achromatic and chromatic mechanisms. Attention, Perception, \& Psychophysics, 71(2), 403-411.

Hsieh, P. J., Colas, J. T., \& Kanwisher, N. (2011). Pop-out without awareness: Unseen feature singletons capture attention only when top-down attention is available. Psychological Science, 22(9), 1220-1226.

Jacob, J., Breitmeyer, B. G., \& Treviño, M. (2013). Tracking the first two seconds: three stages of visual information processing? Psychonomic Bulletin \& Review, 20(6), 1114-1119.

Kang, M. S., Blake, R., \& Woodman, G. F. (2011). Semantic analysis does not occur in the absence of awareness induced by interocular suppression. Journal of Neuroscience, 31(38), 13535-13545.

Lunghi, C., Binda, P., \& Morrone, M. C. (2010). Touch disambiguates rivalrous perception at early stages of visual analysis. Current Biology, 20(4), R143-R144.

Lunghi, C., Morrone, M. C., \& Alais, D. (2014). Auditory and tactile signals combine to influence vision during binocular rivalry. Journal of Neuroscience, 34(3), 784-792.

Maruya, K., Yang, E., \& Blake, R. (2007). Voluntary action influences visual competition. Psychological Science, 18(12), 1090-1098.

Mudrik, L., Faivre, N., \& Koch, C. (2014). Information integration without awareness. Trends in Cognitive Sciences, 18(9), 488-496.

Peirce, J. W. (2007). PsychoPy—psychophysics software in Python. Journal of Neuroscience Methods, 162(1), 8-13.

Railo, H., Salminen-Vaparanta, N., Henriksson, L., Revonsuo, A., \& Koivisto, M. (2012). Unconscious and conscious processing of color rely on activity in early visual cortex: a TMS study. Journal of Cognitive Neuroscience, 24(4), 819-829.

Ro, T., Singhal, N. S., Breitmeyer, B. G., \& Garcia, J. O. (2009). Unconscious processing of color and form in metacontrast masking. Perception \& Psychophysics, 71(1), 95-103.

Sakuraba, S., Sakai, S., Yamanaka, M., Yokosawa, K., \& Hirayama, K. (2012). Does the human dorsal stream really process a category for tools? Journal of Neuroscience, 32(11), 3949-3953.

Salomon, R., Lim, M., Herbelin, B., Hesselmann, G., \& Blanke, O. (2013). Posing for awareness: Proprioception modulates access to visual consciousness in a continuous flash suppression task. Journal of Vision, 13(7), 2-2.

Schmidt, T. (2000). Visual perception without awareness: Priming responses by color. In T. Metzinger (Ed.), Neural correlates of consciousness (pp. 157-170). Cambridge: MIT Press.

Schmidt, T. (2002). The finger in flight: Real-time motor control by visually masked color stimuli. Psychological Science, 13(2), 112-118.

Soto, D., Mäntylä, T., \& Silvanto, J. (2011). Working memory without consciousness. Current Biology, 21(22), 912-913.

Symes, E., Tucker, M., Ellis, R., Vainio, L., \& Ottoboni, G. (2008). Grasp preparation improves change detection for congruent objects. 
Journal of Experimental Psychology: Human Perception and Performance, 34(4), 854.

Tapia, E., Breitmeyer, B. G., \& Shooner, C. R. (2010). Role of taskdirected attention in nonconscious and conscious response priming by form and color. Journal of Experimental Psychology: Human Perception and Performance, 36(1), 74.

Townsend, J. T., \& Ashby, F. G. (1978). Methods of modeling capacity in simple processing systems. Cognitive theory, 3, 200-239.

Townsend, J. T., \& Ashby, F. G. (1983). Stochastic modeling of elementary psychological processes. Cambridge: Cambridge University Press.

Tsuchiya, N., \& Koch, C. (2005). Continuous flash suppression reduces negative afterimages. Nature Neuroscience, 8(8), 1096.

Tulving, E. (2002). Episodic memory: From mind to brain. Annual Review of Psychology, 53, 1-25.
Wang, F., Sun, J., Sun, P., Weidler, B. J., \& Abrams, R. A. (2017). Influence of simple action on subsequent manual and ocular responses. Attention, Perception, \& Psychophysics, 79(2), 389-395.

Weidler, B. J., \& Abrams, R. A. (2014). Decomposing the action effect: How simple actions affect subsequent perception. Attention, Perception, \& Psychophysics, 76(4), 1242-1252.

Weidler, B. J., \& Abrams, R. A. (2017). Simple actions influence pop-out search. Visual Cognition, 1-14.

Wykowska, A., Schubö, A., \& Hommel, B. (2009). How you move is what you see: action planning biases selection in visual search. Journal of Experimental Psychology: Human Perception and Performance, 35(6), 1755.

Yang, E., Brascamp, J., Kang, M. S., \& Blake, R. (2014). On the use of continuous flash suppression for the study of visual processing outside of awareness. Frontiers in Psychology, 5. 